\title{
CD4 Positive
}

National Cancer Institute

\section{Source}

National Cancer Institute. CD4 Positive. NCI Thesaurus. Code C153220.

An indication that CD4 expression has been detected in a sample. 